in 1842 . In the first forty years only eight or ten cases were recorded, but in the last ten years a large number of cases have been reported. There are now in the Philadelphia Hospital five or six typical cases of chronic adult chorea, most of which are of the hereditary type.

The PRESIDENT.-I would ask Dr. Sinkler what connection he thinks that there may be between this class of cases and those which occur in pregnancy. The latter cases sometimes run a chronic course and present exactly this appearance. They have no hereditary history. They begin as an acute essential chorea and sometimes pass into this chronic form, persisting after confinement. I remember one case which ran into insanity. Another case has been choreaic for five years. I would also ask Dr. Sinkler if he has observed mental derangement as a common sequela of Huntingdon's chorea.

Dr. WharTon Sivkler.-I do not think that we can distinguish clinically between cases of chronic chorea of pregnancy and hereditary chorea. The symptoms are almost identical. I believe that pathologically we shall find the same changes, evidently some cortical changes. These forms of chorea are due to organic change, differing in that respect from Sydenham's chorea, and the cortex is undoubtedly the seat of disease as has been shown by several post-mortem examinations.

Speaking of the chronic chorea of pregnancy I recall an interesting case in a young woman in whom the disease continued for several years. She became pregnant and the movements were greatly exaggerated, but after delivery the movements ceased and she has been well ever since.

In answer to Dr. Lloyd's question, I would say that nearly all cases of Huntington's chorea terminate in dementia.

Dr. Wharton Sinkler read a paper on

\title{
NERVE SUTURE.
}

He presented a patient in whom an operation for suture of the musculo spiral nerve was successfully performed three months after complete section of the nerve. The patient was a man, twenty-six years of age, married, intemperate as to the use of stimulants. In the early part of February, I894, he was stabbed with a small blade of a penknife just above the left elbow. The 
musculo-spinal nerve was severed, and immediately fol lowing the injury there was complete loss of motion in the extensors of the wrist and fingers. There was a sensation of formication in the thumb and forefinger for a day or two after the injury, but this soon subsided, and no loss of sensation was noted. The patient was first seen by Dr. Sinkler about ten weeks after the division of the nerve. At that time there was complete wristdrop of the left hand, and inability to extend any of the fingers. The hand was much swollen, and on the dorsum of the hand was the hump which is characteristic of extensor paralysis. The distal phalanges of the fingers could be extended by the interossei muscles. There was no loss of power in the flexors, but owing to paralysis of the antagonist muscles there was inability to flex the fingers with any strength, so that the patient could not move the dynamometer one degree. There was no loss of tactile sensation, with the exception of a small area on the posterior and inner aspect of the thumb extending from the carpo-metacarpal articulation of the last phalangeal joint. In this region sensation was impaired, but not entirely lost. The muscles of the forearm and the first interosseous were wasted, and gave no response to the strongest faradic current that could be borne; to the galvanic current the reaction of degeneration was present. The hand muscles responded normally to the electricity.

On May 5 th, three months after the injury, Dr. W. W. Keen cut down upon the musculo-spiral nerve and united the severed ends. The nerve was found to be markedly bulbous at the point at which it had been severed, the bulb apparently consisting of connective tissue. The bulbous portion was excised, and the two extremities stretched by the thumb and finger, and elongated sufficiently to bring them into opposition. The two ends were then sutured with two fine silk threads.

The excised portion of the nerve was examined by Dr. D. Braden Kyle, who reported that he found the severed portion of the nerve about three-fourths of an inch in its greatest diameter and one-half of an inch in its smallest diameter, the greatest diameter being in line with the trunk of the nerve. Microscopically the bulbous portion of the tissue appeared more fibrous than the portion of the trunk attached. On section microscopically it presented the same appearance. Under the micro- 
scope, above and below the bulbous portion, the tissue presented much the normal appearance, except its reaction to stain, the cells seeming to be swollen or undergoing some infiltrating process, the nerve-sheath being thickened. In the bulbous portion partial longitudinal section of nerve filaments could be demonstrated, but the main part of the bulbous portion was composed of fibrous connective tissue. Distinct nerve fibres were present in the bulbous portion, but these fibres were widely disseminated, and were not arranged in bundles.

On May 28th, two weeks after the operation, an examination of the patient was made. There was no change in the paralyzed muscles, and the electrical reactions were the same as they had been in the previous examination, which was made on April isth. 'The use of galvanism was begun, and steadily persisted in, with the addition of massage later. There was no evidence of return of power until the latter part of October, five months after the operation. At this time the patient began to have slight power to extend the wrist. There was gradual restoration of power, and on December $26 \mathrm{th}$, that is, seven months after the operation, the patient was able to extend the wrist fully and strongly. There was full power of extension of all of the fingers. With the dynamometer the grip of the right hand was I4O, with the left 65. At this time the extensor muscles of the left forearm still failed to respond to the faradic current, and with the galvanic current the anodal closing contraction was about equal to the kathodal closing contraction. A strong current was necessary, however, to cause the muscular contractions.

On April 9, I 895, an examination shows an increase in the muscular power, and an interesting change in the electrical reactions. The muscles still fail to respond to the faradic current, but there is also loss of reaction to the galvanic current with the strongest current avail. able. A very strong galvanic current causes slight tetanic contractions of the extensor carpi radialis.

Dr. Sinkler remarked that the case was interesting for a number of reasons. First, because it showed the advantage of nerve suture after an interval of three months, and the importance of thorough and long-con. tinued electrical treatment to the nerve and paralyzed muscles, even when but little encouragement had been obtained after several months treatment. The electrical reactions are also important. When the patient was first 
seen, the reaction of degeneration and complete absence of response to the faradic current showed that there was entire division of the nerve, and the fact that restoration of voluntary power before the return of the normal electrical reactions is interesting, because it bears out the experience of those observers who have given detailed accounts of cases after nerve suture. A point of especial in terest is the effort which had been made in regeneration of the nerve. The fact of nerve filaments being present in the excised bulbous portion showed that the process of regeneration had begun, and it seems probable that in time that this process would have become more fully developed, and possibly have restored the function of the nerve.

\section{DISCUSSION.}

Dr. JOHN K. MrTCHELL.-In the last two years I have seen fifty-five cases of section of nerves, some sutured and some not, and I have examined the notes of over two. hundred and fifty cases of sectioned nerves with more or less complete recovery. I deny the possibility of prim. ary union. I believe the cases that have been reported as instances of primary union have been cases where sensation alone has been studied and not motion. The return of sensation isas depended upon anastomsis and not upon reunion of the out-nerve.

The most interesting practical point is the recognition of the great success of secondary suture in this case. I believe that secondary suture, even at a much later period should be tried and that it would many times be successful. I think, too, that in favorable cases motion will be found to return as soon after secondary suture as after primary suture. The degeneration which follows every section necessarily occupies many weeks, and these weeks might as well pass without the nerve being united.

Dr. Wharton Sinkler.-The time which intervened in this case between the injury and the suturing was only three months, which is only a moderate length of time. Cases have been reported where longer intervals have elapsed. In one case the sciatic nerve was united nine months after its division by a scythe and there was complete restoration of function.

Adjourned. 